\title{
Sulfato de atropina nos parâmetros hemodinâmicos e hemogasométricos de cães anestesiados com clorpromazina, dexmedetomidina e isoflurano
}

\author{
Hemodynamic and hemogasometric in the atropine administration in dogs anesthetized with \\ chlorpromazine and dexmedetomidine and isoflurane
}

\author{
Fabíola Niederauer Flôres ${ }^{\mathrm{I}}$ Aury Nunes de Moraes $^{\mathrm{I}}$ Nilson Oleskovicz ${ }^{\mathrm{I}}$ Flávia de Oliveira $^{\mathrm{II}}$ \\ Neida Bortoluzzi ${ }^{\mathrm{II}}$ Vanessa Minsky ${ }^{\mathrm{II}}$ André Soares ${ }^{\mathrm{III}}$
}

\section{RESUMO}

Seis cães, pesando $17,9 \mathrm{~kg} \quad( \pm 3,9)$, foram anestesiados em duas ocasiões, com intervalo de sete dias, obedecendo estudo cego. A indução e a manutenção anestésica foram realizadas com isoflurano em ventilação mecânica. Depois da instrumentação, a concentração final de isoflurano foi fixada em 1,3V\% durante o estudo. Após período de estabilização de 30 minutos, foram mensurados os parâmetros hemodinâmicos e hemogasométricos (M-15); na seqüência, administrou-se atropina (grupo atropina) ou cloreto de sódio 0,9\% (grupo salina). Passados 15 minutos, os parâmetros foram avaliados novamente (M0) e aplicou-se clorpromazina e dexmedetomidina (Clor-Dex). As variáveis foram mensuradas por mais 65 minutos depois da Clor-Dex. Empregou-se ANOVA de uma via e teste Student Newman Keuls para valores entre tempos dentro de cada grupo e teste t pareado para avaliação das médias entre grupos $(P \leq 0,05)$. A freqüencia cardíaca foi maior no grupo atropina depois da administração de ClorDex, e houve redução nos valores de Índice Cardíaco (IC) em ambos os grupos. Embora os valores médios de IC tenham sido maiores no grupo atropina, não houve diferença significativa entre grupos administração de Clor-Dex promoveu aumento da pressão arterial em cães tratados com atropina. A pressão arterial média (PAM) foi significativamente maior no grupo atropina dos 5 aos 65 minutos após administração de Clor-Dex, assim como o Índice de Resistência Periférica Total (IRPT) aumentou em ambos os grupos depois da Clor-Dex.. Os parâmetros hemogasométricos não apresentaram diferenças significativas. A administração de atropina antes da clorpromazina e da dexmedetomidina resultou em aumento da pressão arterial. A bradicardia induzida pela administração da associação de fármacos foi prevenida pelo anticolinérgico, porém a redução do débito cardíaco não foi prevenida.

Palavras-chave: atropina, dexmedetomidina, clorpromazina, cães.

\section{ABSTRACT}

Six dogs weighing $17.9 \pm 3.9 \mathrm{~kg}$ were anesthetized on two occasions, at least 7 days apart, in randomized blinded manner. Anesthesia was induced and maintained with isoflurane in mechanical ventilation. After instrumentation, the end-tidal isoflurane was maintained at $1,3 \% \mathrm{~V}$ throughout the study. After a 30 minutes stabilization period ( $M-15)$, baseline hemodynamic parameters and arterial blood gases were recorded and atropine (atropine group) or $0.9 \% \mathrm{NaCl}$ (saline group) were administered. Fifteen minutes later, data were recorded again (MO) and a chlorpromazine-dexmedetomidine (Chlor-Dex) combination was administered. Variables were measured for an additional 65 minutes after Chlor-Dex. A one-way ANOVA-Student-Newman-Keuls was used for comparisons within groups, while a paired test was used for comparisons between groups $(P \leq 0,05)$. Heart rate was higher in atropine group after Chlor-Dex administration. Cardiac index (CI) was reduced from baseline after Chlor-Dex in both treatments. Although mean CI values tended to be higher in atropine group, CI did not differ between groups. Chlor-Dex administration caused increased arterial blood pressure in dogs treated with atropine. Mean arterial pressure (MAP) was significantly higher in the atropine group from 5 to $65 \mathrm{~min}$ after Chlor-Dex. The systemic vascular resistance index (SVRI) increased from baseline in both groups after Chlor-Dex administration. No significant differences were observed for arterial blood gases. Atropine administration prior to ChlorDex resulted in increased arterial blood pressure. Bradycardia induced by the administration of these drugs was prevented by the anticholinergic given, however decrease in cardiac output was not prevented.

Key words: atropine, dexmedetomidine, chlorpromazine, dogs.

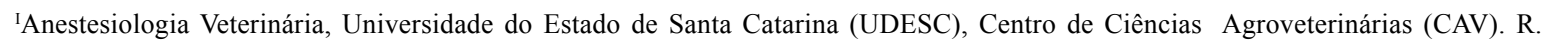

Walmor Ribeiro, 514, ap.207, Coral, Lages, SC, Brasil. E-mail: fanf_vet@yahoo.com.br. *Autor para correspondência.

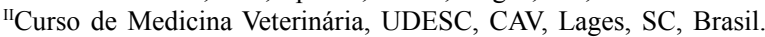

IIIPrograma de Pós-graduação em Ciências Veterinárias, UDESC, CAV, Lages, SC, Brasil. 


\section{INTRODUÇÃO}

A associação de fármacos injetáveis com a anestesia inalatória tem sido proposta com o objetivo de reduzir a CAM do anestésico inalatório, além de prevenir efeitos indesejáveis possivelmente observados com o emprego de um agente único.

A administração prévia de anticolinérgicos tem sido recomendada para prevenir os efeitos cardiovasculares de outros fármacos, como os dos agonistas alfa-2 adrenoceptores, que provocam bradicardia e arritmias cardíacas (ALIBHAI et al., 1996; KO et al., 2001; SINCLAIR et al., 2002). Porém, segundo BLOOR etal.(1992),ALIBHAI etal.,(1996) e SINCLAIR et al. (2002), isso agrava e prolonga o aumento da pressão arterial produzida pelo agonista alfa-2 adrenoceptor em associação com taquicardia.

A clorpromazina é um derivado fenotiazínico capaz de provocar o relaxamento da musculatura lisa vascular, produzindo diminuição da resistência vascular periférica, bem como, depressão nos centros bulbares cardiovascular e respiratório (BALDESSARINI, 1996). Conforme SASADA \& SMITH (1997), ocorre hipotensão por bloqueio alfa-adrenérgico periférico e parte por ação central, levando ao desenvolvimento de taquicardia reflexa.

A dexmedetomidina é o mais recente fármaco agonista alfa- 2 adrenérgico em uso, apresentando alta seletividade para os receptores alfa-2 (BAGATINI et al., 2002), sendo que seu emprego promove boa estabilidade hemodinâmica (VILLELA \& NASCIMENTO JÚNIOR, 2003). Segundo BAGATINI et al. (2002), a dexmedetomidina tem como característica a redução de outros anestésicos; portanto, conforme MAGALHÃES et al. (2004), seu uso como adjuvante da anestesia geral inalatória proporciona uma interação farmacológica sinérgica, com redução da concentração do anestésico volátil e, dessa forma, maior estabilidade hemodinâmica do paciente.

A dexmedetomidina produz efeitos cardiovasculares semelhantes aos demais agonistas alfa-2 adrenérgicos (TALKE et al., 2000; BAGATINI et al., 2002), não promovendo depressão respiratória importante(VILLELA \& NASCIMENTO JÚNIOR, 2003) ou depressão nos valores de gases sangüíneos (TAKROURI et al., 2002).

$\mathrm{O}$ isoflurano provoca depressão cardiovascular, pulmonar e das funções neuronais de maneira dose-dependente, não reduzindo significativamente o débito cardíaco (PADDLEFORD, 2001). Além disso, promove diminuição da resistência vascular, reduzindo a pressão sangüínea arterial (SASADA \& SMITH, 1997).
Devido à escassez de informações sobre as associações anestésicas propostas neste estudo, objetivou-se avaliar os efeitos hemodinâmicos e hemogasométricos da pré-medicação com atropina em cães tratados com clorpromazina e dexmedetomidina sob anestesia geral com isoflurano. Pretendeu-se, ainda, avaliar a capacidade do anticolinérgico em prevenir a bradicardia produzida pelo alfa-2 adrenoceptor, assim como sua implicação direta na resistência vascular periférica, e, ao mesmo tempo, avaliar a capacidade do fenotiazínico em atenuar o aumento da pressão arterial produzida pelo fármaco agonista alfa-2 adrenérgico.

\section{MATERIAL E MÉTODOS}

Foram utilizados seis cães hígidos, machos ou fêmeas, sem raça definida, pesando $17,9 \mathrm{~kg}( \pm 3,9)$, oriundos de doações. Os animais foram submetidos ao protocolo experimental duas vezes, com intervalo mínimo de sete dias entre os tratamentos, sendo que os seis animais $(n=6)$ passaram pelos dois grupos estudados (Grupo Salina e Grupo Atropina), obedecendo estudo cego.

Após jejum alimentar de 12 horas e hídrico de seis horas, os animais foram induzidos a anestesia geral com isoflurano ${ }^{\mathrm{a}}$ administrado através de máscara facial, sendo intubados e a anestesia mantida em ventilação espontânea com isoflurano em CAM adequada ao procedimento de instrumentação necessária ao experimento, com sensor do analisador de $_{\text {gases }}{ }^{\mathrm{b}}$ adaptado à extremidade da sonda orotraqueal conectada ao circuito anestésico. A instrumentação constou de canulação da artéria tibial dorsal esquerda, para mensuração da pressão arterial e para coletas de sangue arterial, além da introdução do cateter de Swan $\mathrm{Ganz}^{\mathrm{c}}$ através da veia jugular direita e posicionamento na luz da artéria pulmonar para avaliação dos demais parâmetros hemodinâmicos.

O correto posicionamento do cateter de Swan Ganz foi confirmado pela leitura do traçado da onda de pulso no monitor do equipamento multiparamétrico ${ }^{d}$ empregado para mensuração dos parâmetros hemodinâmicos. Os parâmetros hemogasométricos ${ }^{\mathrm{e}}$ foram avaliados através da análise de amostras de sangue coletadas da artéria tibial dorsal em seringas heparinizadas.

Ao término da instrumentação, os animais foram mantidos em ventilação mecânica com volume corrente de $15 \mathrm{~mL} \mathrm{~kg}^{-1}$, no modo ciclado a volume, mantendo freqüência respiratória de 12 movimentos por minuto, com $\mathrm{ETCO}_{2}$ entre 35 a $45 \mathrm{mmHg}$ e a concentração do isoflurano tendo sido reduzida para 
1,3V\%. Transcorridos 30 minutos do período de estabilização hemodinâmica, foi dado início ao protocolo experimental, obedecendo, por meio de estudo cego, aos seguintes tratamentos:

GAtropina $(\mathrm{n}=6)$ : Atropina ${ }^{\mathrm{f}}\left(0,04 \mathrm{mg} \mathrm{kg}^{-1}\right.$ intramuscular - IM) + Clorpromazina ${ }^{9}\left(0,5 \mathrm{mg} \mathrm{kg}^{-1}\right.$ intravenosa - IV) + Dexmedetomidina ${ }^{\mathrm{h}}\left(3 \mu \mathrm{g} \mathrm{kg}^{-1} \mathrm{IV}\right) \mathrm{e}$;

GSalina ( $n=6$ ): Cloreto de $\mathrm{Na} 0,9 \%$ (volume equivalente a atropina IM) + Clorpromazina $\left(0,5 \mathrm{mg} \mathrm{kg}^{-1}\right.$ IV) + Dexmedetomidina $\left(3 \mu \mathrm{g} \mathrm{kg}^{-1} \mathrm{IV}\right)$

Durante o experimento, foi fornecida a fluidoterapia com Ringer lactato $\left(10 \mathrm{~mL} \mathrm{~kg}^{-1} \mathrm{~h}^{-1}\right)$ e a temperatura corporal dos cães foi mantida por meio de bolsas aquecidas e controle da temperatura ambiente.

A mensuração das variáveis iniciou-se logo após o período de estabilização hemodinâmica (M-15). Na seqüência, aplicou-se atropina (grupo atropina) ou cloreto de $\mathrm{Na}$ 0,9\% (grupo salina) via IM. Passados 15 minutos, avaliou-se M0 e administrou-se clorpromazina e dexmedetomidina (Clor-Dex) em seringas separadas e em 60 segundos cada uma via IV, iniciando-se pela clorpromazina. Cinco minutos após Clor-Dex, mensurou-se M5. As aferições seguintes (M20, M35, M50, M65) foram realizadas em intervalos de 15 minutos, a partir de M5, totalizando 65 minutos de avaliação após administração IV da associação de clorpromazina e dexmedetomidina.

Os parâmetros avaliados foram: freqüência cardíaca (FC), saturação de oxihemoglobina (SatO2), pressão arterial sistólica, média e diastólica (PAS, PAM, PAD), pressão venosa central (PVC), débito cardíaco (DC), índice cardíaco (IC), volume e índice sistólico (IS), pressão da artéria pulmonar (PAP), pressão de oclusão da artéria pulmonar (POAP), trabalho e índice do trabalho ventricular esquerdo (ITVE), resistência e índice da resistência periférica total (IRPT), freqüência respiratória $(f)$, volume corrente $(\mathrm{VC})$, volume minuto (VM), $\mathrm{CO}_{2}$ no final da expiração $\left(\mathrm{ETCO}_{2}\right)$, pressão parcial de oxigênio no sangue arterial $\left(\mathrm{PaO}_{2}\right)$, pressão parcial de dióxido de carbono no sangue arterial $\left(\mathrm{PaCO}_{2}\right)$, bicarbonato de sódio $\left(\mathrm{HCO}_{3}\right)$, déficit de base (DB), potencial de hidrogênio $(\mathrm{pH})$, concentrações de sódio $(\mathrm{Na})$, potássio $(\mathrm{K})$, hemoglobina arterial $(\mathrm{Hb})$ e temperatura corporal $(\mathrm{T})$.

A análise estatística foi realizada através do programa computacional Sigma Stat for Windows versão 3.0.1, SPSS Inc. 2003. Para avaliação das médias entre grupos dentro de cada tempo, os dados coletados foram submetidos ao teste $\mathrm{T}$ pareado $(\mathrm{P}=0,05)$. Para avaliação das médias entre tempos, dentro de cada grupo, os dados foram submetidos à análise de variância de uma via (ANOVA) e, para a avaliação das diferenças encontradas, utilizou-se o Teste de Student Newman Keuls $(\mathrm{P} \leq 0,05)$.

\section{RESULTADOS}

Os resultados obtidos no presente estudo, com a avaliação dos parâmetros hemodinâmicos, encontram-se dispostos na tabela 1 e na figura 1 .

Os valores médios de FC foram menores no grupo salina a partir de M5 até M50, apresentando diferença estatística em relação ao grupo atropina. $\mathrm{O}$ grupo salina também apresentou diferença estatística entre o valor médio de FC do M5 em relação ao M-15 (Figura 1A).

Os valores referentes a PAS, PAD, PAM apresentaram-se superiores no grupo atropina, a partir de M5, em relação ao grupo salina, tendo este aumento de pressão arterial permanecido durante todo o tempo estudado. Evidenciaram-se valores médios de PAM estatisticamente diferentes do M-15, em M5 e M20 no grupo salina, e em M5, M20, M35, M50 do grupo atropina (Figura 1B).

Débito e índice cardíaco apresentaram-se significantemente reduzidos em M5 em ambos os grupos, em relação ao M-15 (Figura 1C). Nos valores médios de IS, houve decréscimo a partir de M5 até o M35 no grupo atropina em relação ao M-15 (Figura 1D). Na PVC, observou-se aumento em M5 no grupo salina em relação ao M-15.

A PAP e o POAP estiveram aumentadas no grupo atropina em M5, em relação ao M-15, no grupo salina, a POAP apresentou aumento em M5 e M20, em relação ao M-15. Observou-se, embora não estatisticamente significativo, aumento nos valores de PAP e POAP, a partir de M5 até M50, no grupo atropina em relação ao grupo salina.

No ITVE, o M20 do grupo atropina apresentou-se significativamente superior ao M20 do grupo salina (Figura 1E), porém observa-se que, embora não-significativo, a partir de M0 os valores de ITVE apresentaram-se aumentados em relação aos valores apresentados no grupo salina.

Nos valores de IRPT, evidenciou-se aumento a partir de M5 em ambos os grupos, sendo mais acentuado e duradouro no grupo atropina (Figura 1F). Os parâmetros hemogasométricos não apresentaram diferença significativa e a ventilação mecânica promoveu estabilidade nos parâmetros ventilatórios.

\section{DISCUSSÃO}

Neste estudo, o pré-tratamento com atropina estabilizou a FC, evitando a bradicardia característica da dexmedetomidina (BEIER et al., 2003). Os agonistas alfa-2 adrenoceptores, segundo diversos autores 
Tabela 1 - Efeitos da administração de clorpromazina e dexmedetomidina sobre os parâmetros hemodinâmicos de cães pré-tratados ou não por atropina e submetidos a anestesia com isofluorano sob ventilação mecânica, representação de médias e erros-padrão da média (EPM), em cada momento da avaliação.

\begin{tabular}{|c|c|c|c|c|c|c|c|c|}
\hline \multirow{2}{*}{ Variável } & & \multicolumn{7}{|c|}{ - } \\
\hline & & M-15 & M0 & M5 & M20 & M35 & M50 & M65 \\
\hline \multirow[t]{2}{*}{ FC } & GS & $96 \pm 7$ & $93 \pm 8$ & $59 * \pm 6$ & $64 \pm 3$ & $70 \pm 3$ & $71 \pm 2$ & $76 \pm 3$ \\
\hline & GA & $98 \pm 6$ & $104 \pm 8$ & $108^{\#} \pm 4$ & $105^{\#} \pm 4$ & $99^{\#} \pm 5$ & $92^{\#} \pm 5$ & $90 \pm 4$ \\
\hline \multirow[t]{2}{*}{$\mathrm{IC}$} & GS & $3,4 \pm 0,6$ & $3,6 \pm 0,6$ & $1,7^{*} \pm 0,2$ & $1,9 * \pm 0,1$ & $2,3 \pm 0,2$ & $2,6 \pm 0,4$ & $2,8 \pm 0,3$ \\
\hline & GA & $3,5 \pm 0,5$ & $4,2 \pm 0,7$ & $1,8^{*} \pm 0,2$ & $2,4 \pm 0,2$ & $2,6 \pm 0,2$ & $2,8 \pm 0,2$ & $3,1 \pm 0,2$ \\
\hline \multirow[t]{2}{*}{ PAM } & GS & $84 \pm 6$ & $82 \pm 5$ & $115^{*} \pm 2$ & $103 * \pm 1$ & $95 \pm 2$ & $87 \pm 4$ & $84 \pm 4$ \\
\hline & GA & $84 \pm 5$ & $84 \pm 6$ & $171^{\# *} \pm 10$ & $148^{\# * \pm 7}$ & $135^{\# *} \pm 7$ & $116^{\# * \pm 6}$ & $103^{\#} \pm 6$ \\
\hline \multirow[t]{2}{*}{$\mathrm{PVC}$} & GS & $10,3 \pm 1,3$ & $8,5 \pm 0,6$ & $14,8^{*} \pm 0,8$ & $12,0 \pm 0,6$ & $11,5 \pm 0,9$ & $10,5 \pm 0,9$ & $10,5 \pm 0,9$ \\
\hline & GA & $8,5 \pm 0,6$ & $7,5 \pm 1,3$ & $10,8 \pm 2,0$ & $9,8 \pm 0,8$ & $8,3 \pm 1,0$ & $8,6 \pm 0,9$ & $8,0 \pm 1,4$ \\
\hline \multirow[t]{2}{*}{ IS } & GS & $34,6 \pm 3,8$ & $37,6 \pm 4,5$ & $32,0 \pm 7,7$ & $30,2 \pm 2,8$ & $33,7 \pm 3,7$ & $35,8 \pm 5,0$ & $37,0 \pm 3,2$ \\
\hline & GA & $35,4 \pm 3,4$ & $39,5 \pm 3,3$ & $16,9^{*} \pm 1,4$ & $22,5^{*} \pm 1,5$ & $26,3^{*} \pm 1,9$ & $30,9 \pm 1,8$ & $34,5 \pm 2,1$ \\
\hline \multirow[t]{2}{*}{ PAP } & GS & $18,8 \pm 1,8$ & $19,2 \pm 1,7$ & $21,7 \pm 1,2$ & $20,0 \pm 0,9$ & $18,7 \pm 0,9$ & $18,3 \pm 0,9$ & $17,7 \pm 0,8$ \\
\hline & GA & $18,7 \pm 1,6$ & $20,5 \pm 1,7$ & $30,5 * \pm 3,3$ & $25,0 \pm 1,7$ & $22,8 \pm 1,8$ & $18,8 \pm 2,1$ & $18,7 \pm 1,0$ \\
\hline \multirow[t]{2}{*}{ POAP } & GS & $11,5 \pm 1,1$ & $13,2 \pm 1,4$ & $18,8 * \pm 0,9$ & $15,7^{*} \pm 0,7$ & $13,7 \pm 0,6$ & $13,3 \pm 0,7$ & $12,5 \pm 0,9$ \\
\hline & GA & $12,7 \pm 1,4$ & $13,0 \pm 1,3$ & $27,7 * \pm 3,2$ & $19,5 \pm 1,4$ & $17,2 \pm 1,3$ & $15,7 \pm 1,1$ & $12,8 \pm 0,9$ \\
\hline \multirow[t]{2}{*}{ ITVE } & GS & $3,8 \pm 0,8$ & $3,8 \pm 0,7$ & $2,5 \pm 0,3$ & $2,5 \pm 0,2$ & $2,9 \pm 0,3$ & $2,9 \pm 0,4$ & $3,1 \pm 0,4$ \\
\hline & GA & $3,9 \pm 0,6$ & $4,5 \pm 0,5$ & $4,1 \pm 0,5$ & $4,7^{\#} \pm 0,6$ & $4,6 \pm 0,5$ & $4,3 \pm 0,4$ & $4,2 \pm 0,4$ \\
\hline \multirow[t]{2}{*}{ IRPT } & GS & $2195 \pm 291$ & $2088 \pm 315$ & $6076 * \pm 841$ & $4419 * \pm 281$ & $3444 \pm 367$ & $3004 \pm 418$ & $2498 \pm 268$ \\
\hline & GA & $2120 \pm 237$ & $1889 \pm 317$ & $7816^{*} \pm 369$ & $5376^{*} \pm 293$ & $4333 * \pm 297$ & $3447 * \pm 156$ & $2823 \pm 144$ \\
\hline
\end{tabular}

Momentos: M-15 (basal); M0 (15 minutos após administração de atropina ou salina); M5 (5 minutos após administração de clorpromazina e dexmedetomidina); M20 (20 minutos após M0); M35 (35 minutos após M0); M50 (50 minutos após M0); M65 (65 minutos após M0).

Variáveis: freqüência cardíaca (FC), índice cardíaco (IC), pressão arterial média (PAM), pressão venosa central (PVC), índice sistólico (IS), pressão da artéria pulmonar (PAP), pressão de oclusão da artéria pulmonar (POAP), índice do trabalho ventricular esquerdo (ITVE), índice da resistência periférica total (IRPT).

GS: grupo salina; GA: grupo atropina.

\# Difere significativamente do Grupo Salina (T pareado [P=0,05]); * difere de M-15 (Student Newman Keuls [P=0,05]).

(BLOOR et al., 1992; ALIBHAI et al., 1996; KO et al., 2001), induzem aumento da pressão arterial seguida de sua diminuição; sendo assim, o anticolinérgico exacerbou o aumento da pressão arterial (ALIBHAI et al., 1996; SINCLAIR et al., 2002), assim como o fenotiazínico, que promove diminuição da pressão arterial (BALDESSARINI., 1996), não amenizou o efeito da dexmedetomidina.

A redução significativa do DC e IC observada é efeito característico do agonista alfa-2 adrenoceptor (BLOOR et al., 1992; KITAHARA et al., 2002; MURRELL \& HELLEBREKERS., 2005) e a administração prévia do anticolinérgico não amenizou significativamente esta redução, visto que ela ocorreu, de forma semelhante, em ambos os grupos, discordando de BLOOR et al. (1992).

$\mathrm{O}$ aumento no valor de PVC observado em M5 no grupo salina evidencia um aumento na pré-carga cardíaca, aumentando o retorno venoso, sobrecarregando o lado direito do coração. Aumentos na PVC foram observados por BLOOR et al. (1992),
SINCLAIR et al. (2002) e KITAHARA et al. (2002), em estudos com agonistas alfa-2 adrenoceptores em cães. No presente estudo, a atropina minimizou os efeitos dos demais fármacos empregados no estudo sobre a pré-carga cardíaca, possivelmente pela elevação de FC proporcionada, que contrabalançaria o aumento do retorno venoso.

A queda do IS no grupo atropina pode ser explicada pelo aumento da FC observado neste grupo. Sendo assim, o emprego de anticolinérgicos não parece ter sido benéfico, concordando com BLOOR et al. (1992), KO et al. (2001) e SINCLAIR et al. (2002), sendo que a função ventricular esquerda fica prejudicada, diminuindo a performance cardíaca em animais tratados com fármacos agonista alfa-2 adrenérgico e anticolinérgico, pois o acréscimo da FC aumenta a demanda de oxigênio pelo miocárdio. VILLELA et al. (2003) e MAGALHÃES et al. (2004) não obtiveram alterações no IS empregando dexmedetomidina em infusão contínua, evidenciando, assim, a estabilidade hemodinâmica promovida pelo fármaco. 

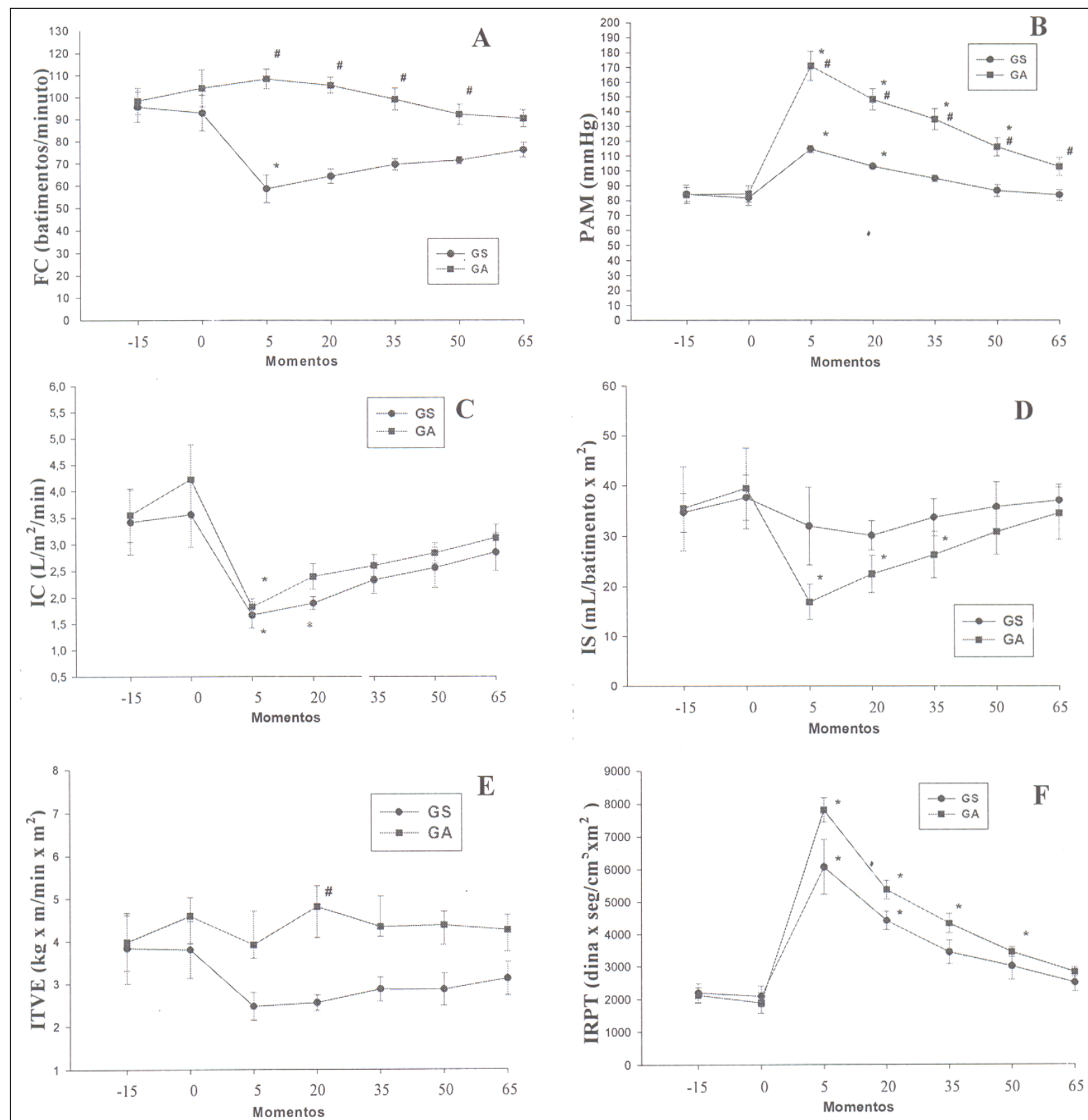

Figura 1 - Representação gráfica dos valores médios de A) Freqüência Cardíaca (batimentos/minuto); B) Pressão Arterial Média $(\mathrm{mmHg})$; C) Índice Cardíaco $\left(\mathrm{L} \mathrm{m}^{-2} \mathrm{~min}^{-1}\right)$; D) Índice Sistólico $\left(\mathrm{mL} /\right.$ batimento $\left.\mathrm{x} \mathrm{m}^{2}\right)$; E) Índice do Trabalho Ventricular Esquerdo ( $\mathrm{kg} \mathrm{x} \mathrm{m/min} \mathrm{x} \mathrm{m}^{2}$ ); F) Índice de Resistência Periférica Total (dina $\mathrm{x} \mathrm{seg} / \mathrm{cm}^{5} \mathrm{xm}^{2}$ ) em cães anestesiados com clorpromazina, dexmedetomidina e isofluorano, pré-tratados ou não por atropina. GS: Grupo Salina; GA: Grupo Atropina. \# Difere significativamente do Grupo Salina (T pareado $[\mathrm{P} \leq 0,05])$; * difere de M-15 (Student Newman Keuls $[\mathrm{P} \leq 0,05])$.

Com relação aos valores médios de PAP e POAP, no grupo atropina, observou-se aumento a partir de M5 até M50 em relação ao grupo salina, embora não diferente estatisticamente, possivelmente pelo aumento de FC e pelo maior trabalho cardíaco provocado pela atropina em contrapartida aos efeitos depressores dos demais fármacos empregados, concordando com BLOOR et al. (1992) e SINCLAIR et al. (2002).
$\mathrm{Na}$ avaliação de ITVE, os valores médios aumentados observados no grupo atropina, que diferem estatisticamente entre grupos no M20 e não diferem estatisticamente a partir de M0 até M65, podem ser explicados pelo aumento na FC promovido pela atropina (ALIBHAI et al., 1996), visto que a FC interfere nos valores de DC (BLOOR et al., 1992), que estão diretamente envolvidos no cálculo de TVE. 
O aumento do IRPT é característica dos agonistas alfa-2 adrenoceptores (BLOOR et al., 1992; SINCLAIR et al., 2002), porém, no grupo atropina, observaram valores superiores, e o aumento de IRPT foi mais duradouro em comparação ao grupo salina. Portanto, há influência do anticolinérgico sobre os valores de IRPT, a atropina exacerbou o aumento de pressão arterial produzida pelo alfa-2, assim como o aumento de IRPT, discordando de SINCLAIR et al. (2002).

\section{CONCLUSÕES}

A atropina mantém a freqüência cardíaca dos animais estável. A dexmedetomidina produz aumento de pressão arterial duradoura, agravada pela administração prévia de atropina. A clorpromazina não ameniza o aumento de pressão arterial produzido pela dexmedetomidina. Portanto, a associação de fármacos proposta não produz alterações significativas nos parâmetros hemogasométricos e no equilíbrio ácidobásico dos animais do estudo.

\section{COMITÊ DE ÉTICA E BEM-ESTAR ANIMAL}

O presente estudo foi aprovado pelo Comitê de Ética e Experimentação Animal (CETEA) do Centro de Ciências Agroveterinárias da Universidade do Estado de Santa Catarina (CAV/UDESC), protocolo 1.28/04, de acordo com legislação vigente e os Princípios Éticos publicados pelo Colégio Brasileiro de Experimentação Animal.

\section{FONTES DE AQUISIÇÃO}

a Isoforine: Cristália Produtos Químicos e Farmacêuticos Ltda Itapira, SP.

b OHMEDA - DTX Plus Pressure Transducer System (Model DT-12) - Singapura.

c CRITICATH SP5105H TD - BD - Becton-Dickinson Singapura.

d Spacelabs Medical Multiparamétrico 90496 - USA.

e Rapidlab 348 - Bayer - São Paulo, SP, Brasil.

${ }^{\mathrm{f}}$ Atropinon - 0,250mg/mL - Hipolabor Farmacêutica LTDA Sabará - MG, Brasil.

g Amplictil - 5mg/mL. Aventis Pharma Ltda. Santo Amaro, SP, Brasil.

h Precedex - 100 $\mu \mathrm{g} / \mathrm{mL}$. Abbott Laboratórios de Brasil Ltda. Rio de Janeiro, RJ, Brasil.

\section{REFERÊNCIAS}

ALIBHAI, H.I.K. et al. Cardiopulmonary effects of combinations of medetomidine hydrochloride and atropine sulphate in dogs. Vet Rec, v.138, p.11-13, 1996.
BAGATINI, A. et al. Dexmedetomidina: farmacologia e uso clínico. Rev Bras Anestesiol, v.52, n.5, p.606-617, 2002.

BALDESSARINI, R.J. Fármacos e o tratamento dos distúrbios psiquiátricos. In: GILMAN, A.G. et al. As bases farmacológicas da terapêutica. 9.ed. Rio de Janeiro: Mc Grow Hill, 1996. p.290-313.

BEIER, S.L. et al Avaliação biespectral e nociceptiva na associação da dexmedetomidina em cães pré-tratados ou não pela atropina e tratados ou não pela buprenorfina. In: ENCONTRO DE ANESTESIOLOGIA VETERINÁRIA, 6., 2003, Passo Fundo/RS. Resumos... Passo Fundo: Universidade de Passo Fundo, 2003. p.26.

BLOOR, B.C. et al. Hemodynamic and sedative effects of dexmedetomidine in dog. J Pharmacol Exp Ther, v.263, n.2, p.690-697, 1992.

KITAHARA, F.R. et al. Efeitos hemodinâmicos da dexmedetomidina em cães. Estudo experimental. Braz J Vet Sci, v.9, n.1, p.128-130, 2002.

KO, J.C.H. et al. Effects of preemptive atropine administration on incidence of medetomidine-induced bradycardia in dogs. $\mathbf{J}$ Amer Vet Med Assoc, v.218, n.1, p.52-58, 2001

MAGALHÃES, E. et al. Relação entre infusão contínua de dexmedetomidina e a fração expirada de sevoflurano monitorada pelo índice bispectral. Rev Bras Anestesiol, v.54, n.3, p.303310,2004

MURRELL, J.C.; HELLEBREKERS, L.J. Medetomidine and dexmedetomidine: a review of cardiovascular effects and antinociceptive properties in the dog. Vet Anaesth Analg, v.32, p.117-127, 2005.

PADDLEFORD, R.R. Manual de anestesia em pequenos animais. 2.ed. São Paulo: Roca, 2001. 423p.

SASADA, M.; SMITH, S. Drugs in anaesthesia and intensive care. 2.ed. New York: Oxford Medical, 1997. 402p

SINCLAIR, M.D. et al. The cardiopulmonary effects of romifidine in dogs with and without prior or concurrent administration of glycopyrrolate. Vet Anaesth Analg, v.29, p.1-13, 2002.

TAKROURI, M.S. et al. Dexmedetomidine in intensive care unit: a study of hemodynamic changes. Middle East J Anesthesiol, v.16, n.6, p.587-595, 2002.

TALKE, P. et al. The hemodynamic and adrenergic effects of perioperative dexmedetomidine infusion after vascular surgery. Anesth Analg, v.90, p.834-839, 2000.

VILLELA, N.R; NASCIMENTO JÚNIOR, P. Uso de dexmedetomidina em anestesiologia. Rev Bras Anestesiol, v.53, n.1, p.97-113, 2003.

VILLELA, N.R. et al. Efeitos cardiovasculares de duas doses de dexmedetomidina. Estudo experimental em cães. Rev Bras Anestesiol, v.53, n.6, p.784-796, 2003. 